\title{
Improving heavy dijet resonance searches using jet substructure at the $\mathrm{LHC}$
}

\author{
Aruna Kumar Nayak ${ }^{1, \mathrm{a}}$, Santosh Kumar Rai ${ }^{2, \mathrm{~b}}$, Tousik Samui ${ }^{2, \mathrm{c}}$ (D) \\ ${ }^{1}$ Institute of Physics, HBNI, Sachivalaya Marg, Bhubaneswar 751 005, India \\ ${ }^{2}$ Regional Centre for Accelerator-based Particle Physics, Harish-Chandra Research Institute, HBNI, Chhatnag Road, Jhusi, \\ Prayagraj (Allahabad) 211 019, India
}

Received: 16 September 2020 / Accepted: 8 January 2021 / Published online: 6 February 2021

(C) The Author(s) 2021

\begin{abstract}
The search for new physics at high energy accelerators has been at the crossroads with very little hint of signals suggesting otherwise. The challenges at a hadronic machine such as the LHC is compounded by the fact that final states are swamped with jets which one needs to understand and unravel. A positive step in this direction would be to separate the jets in terms of their gluonic and quark identities, much in a similar spirit of distinguishing heavy quark jets from light quark jets that has helped in improving searches for both neutral and charged Higgs bosons at the LHC. In this work, we utilise this information using the jet substructure techniques to comment on possible improvements in sensitivity as well as discrimination of new resonances in the all hadronic mode that would be crucial in pinning down new physics signals at HL-LHC, HE-LHC and any future 100 $\mathrm{TeV}$ hadron collider.
\end{abstract}

\section{Introduction}

Resonance search is one of the most simple and direct ways to establish the presence of new particles at a collider. With high centre-of-mass energies available at hadronic colliders such as the Large Hadron Collider (LHC), it serves as a perfect playground for resonance searches of massive particles predicted in many beyond the standard model (BSM) scenarios. The LHC energies and available integrated luminosity can help search for these exotic particles beyond 4-5 TeV. The envisaged $100 \mathrm{TeV}$ hadron machine [1] will expectedly improve this range by nearly a factor of magnitude and more so for strongly interacting particles. However, finding a signal for such strongly interacting heavy particles which dom-

\footnotetext{
a e-mail: nayak@iopb.res.in

be-mail: skrai@hri.res.in

c e-mail: tousiksamui@hri.res.in (corresponding author)
}

inantly decay to either quarks, ${ }^{1}$ gluons or both against the huge QCD background is very difficult. The major challenge at a hadronic machine such as the LHC is compounded by the fact that final states are swamped with jets which one needs to understand and unravel in order to extract signal for new physics in the all hadronic final state. However, there are techniques to reduce these huge QCD backgrounds from the signal. There already exist searches for heavy resonances in the dijet channel by CMS [2-7] and ATLAS [8-11]. The experimental collaborations provide an upper limit at $95 \%$ C.L. on the cross section for heavy resonance production and its decay to three different decay channels, viz. $q q, q g$, and $g g$, separately. Although they do not distinguish between quark or gluon jets explicitly in the final state, they use the line-shape information of the resonance peak to set individual limits [12] for the three different types of final state. In this work, we use jet substructure techniques in studying several exotic resonances in the dijet final state and show that in addition to the line-shape, if we can distinguish between the flavour ${ }^{2}$ of the jets, existing and future limits on the cross section for heavy resonances can be significantly improved. Thus a good knowledge of discrimination between quark and gluon jets will not only help in improving the resonance searches at the HE-LHC and future $100 \mathrm{TeV}$ machine but also help in pinning down the new physics scenario by giving hints on the interaction Lagrangian.

The recent developments in the study of jet substructure provide us with one of the best ways to gather more information from a collider event with large hadronic activity [1317]. The use of jet substructure is becoming popular day by day due to the recent developments in both theoretical and experimental understanding of physics inside the jets, where perturbative and non-perturbative effects dominate in differ-

\footnotetext{
${ }^{1}$ Here quark represents both quark and antiquark.

${ }^{2}$ Here flavour means light quark/antiquark and gluon jets.
} 
Table 1 Examples of resonant particles with the production cross section at $13 \mathrm{TeV}$ LHC for each resonant particle at $2 \mathrm{TeV}$

\begin{tabular}{llll}
\hline Model & Lagrangian & Parameter value & $\sigma_{\text {total }}(\mathrm{fb})$ \\
\hline Triplet diquark & $g_{s} f_{s} \epsilon^{a b c} \phi_{a}^{\dagger} \bar{d}_{b} u_{c}^{C}$ & $f_{s}=0.04$ & 101.1 \\
Coloron & $g_{s} f_{s} G^{\prime a} \bar{u} \gamma^{\mu} T_{a} u$ & $f_{s}=0.09$ & 122.0 \\
Excited quark & $g_{s} \frac{f_{s}}{2 \Lambda} \bar{U}^{*}\left[\gamma^{\mu}, \gamma^{\nu}\right] T_{a} u G_{\mu \nu}^{a}+h . c$. & $\frac{f_{s}}{\Lambda}=1.5 \times 10^{-5} \mathrm{GeV}^{-1}$ & $\frac{f_{s}}{\Lambda}=4.1 \times 10^{-5} \mathrm{GeV}^{-1}, A=0$ \\
Spin-3/2 & $i g_{s} \frac{f_{s}}{\Lambda} \bar{\psi}_{\mu}\left(g^{\mu \nu}+A \gamma^{\mu} \gamma^{\nu}\right) \gamma^{\sigma} T^{a} u G_{\nu \sigma}^{a}+h . c$. & $\kappa=5 \times 10^{-5} \mathrm{GeV}^{-1}$ & 222.3 \\
RS graviton & $\kappa h_{\mu \nu} T^{\mu \nu}$ & $\frac{f_{s}}{\Lambda}=5.8 \times 10^{-5} \mathrm{GeV}^{-1}$ & 179.4 \\
Color octet scalar & $g_{s} \frac{f_{s}}{\Lambda} d_{a b c} \phi^{a} G^{b \mu \nu} G_{\mu \nu}^{c}$ & & 493.9 \\
\hline
\end{tabular}

ent regions in the energy scales. It has been shown that theoretical understanding about the substructure of a jet can even allow us to differentiate between quark-initiated and gluoninitiated jets to a certain extent [18-29] due to the difference in their radiation pattern inside the jet. Further improvements in this direction can be possible with the use of machine learning techniques which along with the substructure picture of the jets makes the discrimination even more robust and allows us to enhance the discrimination power between quark and gluon jets [30-37]. This quark and gluon tagging can therefore be used to improve the search for resonances in several BSM physics scenarios at the colliders.

While discrimination between a quark and a gluon jet would perhaps be a novel approach to identify certain BSM scenarios, in this work we take a slightly different approach. Instead of distinguishing a quark jet from a gluon jet on a jet-by-jet basis, we take an event containing several jets as a whole and the analysis is done on the event variables as well as on the jet substructure observables. The jet substructure observables chosen for quark-gluon discrimination will have different probability distributions for quark and gluon jets. In general, all the jets in a set of events produced at the collider are not entirely quark-initiated or gluon-initiated jets, but a certain fraction of them are quark jets or gluon jets contributing to the events. If these fractions are different for signal events from the background events, there will be some degree of distinguishing power between signal and background. Through this work, we try and show that even if we do not tag the jets in a particular event as quark or gluon jets, we can still conclude with some confidence on whether the event is a signal-like event or a background-like event.

The fraction of quark or gluon jets in a set of events, which we talked about in the last paragraph, can be a fixed fraction generated in every single event or it can be an overall fraction from a set of events. For example, if a strongly interacting heavy fermion (e.g. excited quarks $q^{*}$ ) decays to two lightflavoured jets, then one expects that there will be $50 \%$ quark jets and $50 \%$ gluon jets in each event and hence the fraction will be $50 \%$ in the whole set of events for both quark and gluon jets. On the other hand, if a heavy coloured boson (e.g. a coloron) decays to two light-flavoured jets then it will either decay to a pair of quark jets or to a pair of gluon jets. Hence the overall fraction of quark jets and gluon jets in the collection of events will be determined by the relative coupling of the heavy boson with a quark pair and a gluon pair. However, note that such a fraction in the events could also originate from non-resonant dijet or multijet production processes at a collider. In fact, the Standard Model (SM) dijet background is a non-resonant production of two light flavoured jets. The probability distribution for jet substructure observables will nearly be the same for a given fraction irrespective of whether it originates from every single event or from a collection of events. Thus closer scrutiny of the events with jet tagging, comparing quark-initiated and gluon-initiated jets would be crucial in understanding the heavy resonances.

In this work, we highlight the improvements that can be achieved in dijet resonant searches by the use of jet substructure techniques. Similar studies have been done previously, where in Ref. [38] the jet substructure observables have been suggested to improve the searches while Refs. [39,40] use color discriminant variable and jet energy profile as the means of improving the sensitivity in the dijet channel. However, all three studies considered parton showered events only. Our work is more along the lines presented in Ref. [38] but with substantial improvements in the analysis. We include the SM QCD background and use a fast detector simulation and account for the correction to jet energy scales in the computation of the event variables, similar to the procedure followed by experimental collaborations. Moreover, we use an additional set of observables which are considered to be useful in CMS and ATLAS analyses [21-25]. We also show how one can use jet substructure technique and the boosted decision tree (BDT) multivariate method to distinguish different type of resonances in the dijet channel.

\section{Dijet resonances}

In this study we consider three different kind of dijet resonances, each of which gives us one of the three types of dijet signals: $q q, q g$, or $g g$. For resonances leading to $q q$ final state, each of the jets will be quark-like while it will be 
gluon-like for $g g$ final state. On the other hand, for $q g$ final state, its properties will be an admixture of both quark and gluon-like. Hence, if we consider jet substructure observables of the hardest jet (or second hardest jet), we expect their distributions (observables sensitive to jet-types) to be different for these three cases. The distributions for observables of $q q$ will be far apart from those of $g g$ final state while for the $q g$ final state, the distribution is expected to show an overlap with above two.

In Table 1, we list a few important BSM models and their relevant part of Lagrangians which would yield the dijet signal processes in these models, (e.g. see Refs. [41-43]). Note that we have classified the choice of models based on the intrinsic spin of the resonance in the dijet signal. A more comprehensive list of all possible coloured particles that give a dijet resonance at the LHC can be found in Ref. [44]. We also list the production cross sections for the resonant exotic particle at $13 \mathrm{TeV}$ LHC for the specific values of the parameters listed in Table 1 for each model. The parameter values have been chosen such that the cross sections remain below the existing upper limit from the dijet resonance search by CMS [2]. For further analyses, we restrict ourselves to only three specific models (as no significant difference was observed in the current analysis between models where the resonance differed in spin but gave the same dijet final state). We therefore chose the coloron model [4547] for $q q$ resonance, color octet scalar model [48-50] for $g g$ resonance, and excited quark model [51-55] for $q g$ resonance.

\section{Jet substructure observables}

For the jet substructure analysis we first list out the criterion and observables we are interested in. We classify our requirements based on the event variables and therefore we choose only the simple variables, viz. $p_{T}$ and $\eta$ of the leading and sub-leading jet (arranged according to larger transverse momenta) and their angular separation $\Delta R\left(j_{1}, j_{2}\right)=\sqrt{(\Delta \eta)^{2}+(\Delta \phi)^{2}}$ defined between the two jets. For the jet substructure observables, we refer to the two types of observables as pointed out in Ref. [19,23,24], viz. discrete and continuous observables. The most important ones are:

- Particle and charged particle multiplicity inside a jet [19].

- Les Houches Angularity: LHA $=\sum_{i \in J} \frac{p_{T_{i}}}{p_{T_{J}}} \sqrt{\frac{\Delta R(i, J)}{R}}$ $[20,56]$.

- Girth: $g=\sum_{i \in J} \frac{p_{T_{i}}}{p_{T_{J}}}\left(\frac{\Delta R(i, J)}{R}\right)^{2}[19]$.

- Width: $w=\sum_{i \in J} \frac{p_{T_{i}}}{p_{T_{J}}}\left(\frac{\Delta R(i, J)}{R}\right)$ [57-59].
- Two-point energy correlation variables:

$e_{\beta}=\sum_{i>j \in J} \frac{p_{T_{i}} p_{T_{j}}}{p_{T_{J}}^{2}}\left(\frac{\Delta R(i, j)}{R}\right)^{\beta}[60]$.

- $p_{T}^{2}$ weighted jet minor axis $\left(\sigma_{2}\right)$ with respect to the jet axis in $\eta-\phi$ plane $[23,61]$.

- $p_{T} D=\frac{\sqrt{\sum_{i \in J} p_{T_{i}}^{2}}}{\sum_{i \in J} p_{T_{i}}}[23]$.

where $R$ is the radius parameter of the jet and, in the subscripts, $i, j$ represent the constituents of the jet while $J$ represents the jet.

We know from first principle as well as from data collected in collider experiments that radiation pattern inside a gluon jet differs from that of the light-quark jets. Due to the difference in radiation pattern, many jet substructure observables have been proposed in the literature to discriminate between quark and gluon jets. Though our primary aim in this work is not distinguishing a quark jet from a gluon jet, our analysis procedure is still guided by similar jet substructure observables that help in quark-gluon discrimination. The choice of the observables can be explained as follows.

- Gluon fragments more than a quark due to its colour factor (colour factor ratio is $\frac{C_{A}}{C_{F}}=\frac{9}{4}$ ), which results in higher particle multiplicity in a gluon jet compared to a quark jet.

- Charged particle multiplicity is also more in case of gluon jets than in quark jets.

- Gluon fragmentation function is softer than that of a quark, i.e. constituents of gluon jets tend to be softer than a quark jet. This means $p_{T} D \rightarrow 0$ in the case of a gluon jet while $p_{T} D \rightarrow 1$ in case of quark jet.

- Gluon jets are less collimated compared to quark jets. This gives wider radius for gluon jet with respect to quark jet. So, if the shape of a jet is approximated to an ellipse in $\eta-\phi$ plane, a gluon jet tends to have longer minor axis than a quark jet.

- Combination of the last two points also tends to give higher values for girth, width, LHA and two-point energy correlation variables $\left(e_{\beta}\right)$ for gluon jets compared to quark jets.

To illustrate the difference in the distribution of jet substructure observables between quark and gluon jets, in Fig. 1 we show area normalized distributions for the two jet substructure observables, both for quark (green solid) and gluon (blue dashed) jets in $(q q)$ and $(g g)$ subprocesses in SM respectively. The jet substructure observables are calculated from the leading jets in the subprocesses. We can clearly see that the distribution for a quark jet is quite different from that of a gluon jet. However, for a mixed sample of quark and gluon jets, the distribution will be smeared out. The same two 
Fig. 1 Distribution of jet substructure observables for SM $g g$ events (blue dashed), $q q$ events (green solid), and $q g$ events (red dot-dashed). The distributions shown are for a Les Houches Angularity (LHA) and for $\mathbf{b} p_{T} D$
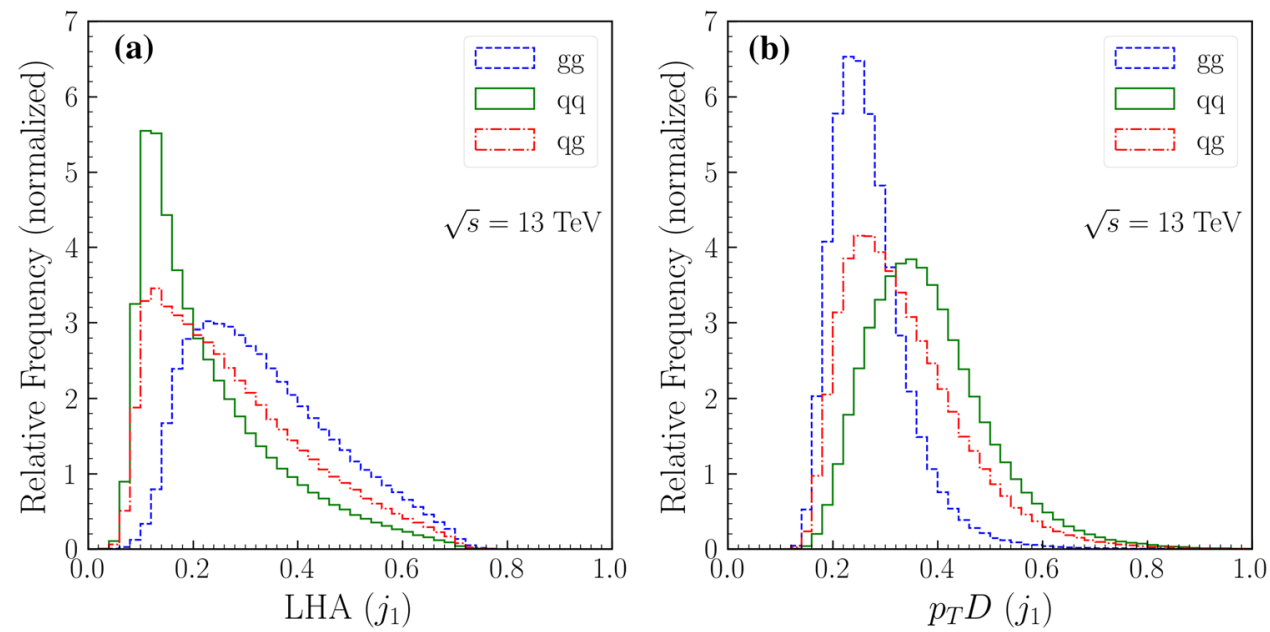

observables are plotted (red dot-dashed) in Fig. 1 for the leading jet in $q g$ events. A selection criteria of $p_{T_{J}}>500 \mathrm{GeV}$ and $|\eta|<2.5$ for both the jets was imposed on the event sample. The anti-kt algorithm with radius parameter $R=0.4$ was used for jet clustering. The distribution of the jet substructure observables shown in Fig. 1 are for Les Houches Angularity (LHA) and for $p_{T} D$. This difference in distribution among quark, gluon and admixture of quark and gluon will form the basis of the analyses to follow.

\section{Analysis and results}

To facilitate this study, Universal FeynRules Object (UFO) [62] files were generated corresponding to the Lagrangian listed in Table 1 using FeynRules 2 . 0 [63,64]. The mass of the heavy resonances have been taken to be $2 \mathrm{TeV}$ for all the three types of resonances. Parton-level dijet final states from the decay of resonant particles were then simulated at $\sqrt{s}=13 \mathrm{TeV}$ using the UFO files with the help of MadGraph5 [65] with a hard $p_{T}$ cut to populate the relevant phase space with enough events. The events generated with $p_{T}>700 \mathrm{GeV}$ for both the jets (parton-level) from MadGraph5 were parton showered and hadronized using Pythia8235[66] with the default tune implemented in Pythia. These showered events were then passed on to Delphes3.4.2[67] for detector simulation. An average of 25 pile-ups have been merged with the generated events inside the Delphes. Jets were clustered using the anti-kt algorithm with radius $R=0.4$ from the Particle Flow outputs from Delphes with the help of FastJet 3.3.2 [68]. In order to reduce the effects of contamination from Underlying Event (UE) and pile-up (PU), many different taggers and groomers have been proposed in the literature e.g. trimming [69], pruning [70,71], mass drop tagger [72] and soft drop [73] groomer. In this work, we use the soft drop groomer which is a IRC safe groomer. After jet clustering, the soft drop groomer was used to groom away the UE and the PU with soft drop parameter $\beta=1.0$ and $z_{\text {cut }}=0.1$. Both the groomed jets from each event are required to have $p_{T}$ more than 700 $\mathrm{GeV}$.

Next, we perform jet energy correction on the groomed jets, i.e. correct the detector-level jet to the particle-level jet following the procedure prescribed in Ref. [74]. We correct the jet four-momenta of the groomed jets by calibrating groomed jets with respect to the Monte Carlo (MC) truth jets. For this purpose, SM dijet samples were generated using MadGraph5 [65] in $p_{T}$ and $\eta$ bins. The MC truth jets were then constructed from the stable particles after showering the dijet events using Pythia8235 [66]. The groomed jets were constructed from the Particle Flow outputs from Delphes following the same procedure described in the previous paragraph. The algorithm and parameters for jet clustering and soft drop groomer were taken to be the same in this case also. The groomed jets have been constructed after merging an average of 25 pile-up event, while no pile-up events have been considered in the case of MC truth jet sample. For the calibration, we imposed a matching condition of $\Delta R<0.25$ between MC truth jets and groomed jets. The events containing at least two matching jets were further considered for the calibration. For each event, we then calculate response as

$R\left(p_{T}^{\text {groomed }}\right)=\frac{p_{T}^{\text {groomed }}}{p_{T}^{\text {truth }}}$

for each of the two jets separately. In each $p_{T}$ and $\eta$ bin, we next compute average of $p_{T}^{\text {groomed }}\left(\overline{p_{T}^{\text {groomed }}}\right)$ and mean response $(\bar{R})$ as a function of $\overline{p_{T}^{\text {groomed }}}$. In each $\eta_{j}$ bin, the response function $\mathscr{R}_{\eta_{j}}\left(p_{T}^{\text {groomed }}\right)$ is obtained from the fit of $\left(\overline{R_{i}}, \overline{p_{T_{i}}^{\text {groomed }}}\right)$ data, where $i$ runs over $p_{T}$ bins. The fitting 


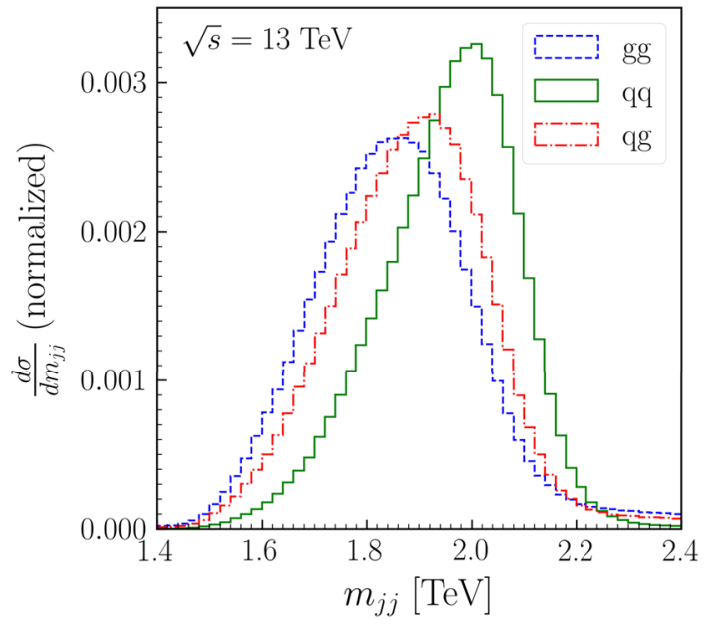

Fig. 2 Distribution of dijet invariant mass for three different types of signals, viz. $g g, q g$ and $q q$ resonance signals

function $\mathscr{R}_{\eta_{j}}$ is parametrized as

$\mathscr{R}_{\eta_{j}}\left(p_{T}^{\text {groomed }}\right)=a_{0}+a_{1} \ln \left(\overline{p_{T}^{\text {groomed }}}\right)$

where $a_{0}$ and $a_{1}$ are fitting parameters. The response function has been computed for leading and sub-leading groomed jets separately. The corrected four momenta $(p)$ of the groomed jets are then obtained by multiplying with the inverse of the response function

$p_{\eta_{j}}^{\text {corrected }}=\frac{p_{\eta_{j}}^{\text {groomed }}}{\mathscr{R}_{\eta_{j}}\left(p_{T}^{\text {groomed }}\right)}$.

With the four momenta corrected groomed jets, we then reconstruct the mass of the heavy resonance by taking the invariant mass of the dijet system. The normalized distribution of dijet invariant mass is plotted in Fig. 2. The dijet invariant mass distribution is one way of looking at the parton content of the jets making up the resonance. It is quite clear that the line-shapes of the resonant mass are different for $q q, q g$, and $g g$ resonances as also mentioned in Ref. [2]. The CMS collaboration has actually used the line shape information to put 95\% C.L. upper bound on the three above mentioned configurations of the dijet resonances [2]. A quick comparison of the line-shape plot with that of Ref. [2] finds that the $m_{j j}$ distributions are in agreement reasonably well. An upper cut on $\left|\eta_{j}\right|<2.15$ for both the jets has been imposed for further analysis. Note that all jet substructure observables have been constructed after the soft drop grooming procedure. However, the four momenta, $p_{T}$ and invariant mass of the dijet system are taken only after jet energy correction.

With the generated events and variables, we then try to find distinguishing score of the three different types of res- onances from the SM background. Since, at a hadron collider, one cannot really avoid SM QCD background, we consider the non-resonant SM dijet as our background in this analysis. Note that for the SM QCD background, we shall use the same tools and parameters discussed earlier. The dijet background has been simulated at the leading order (LO) of QCD. The properties of the leading and subleading jets of the QCD background will again be a mixture of the properties of pure quark and pure gluon jets. So we expect better discrimination for $q q$ and $g g$ resonances from the SM QCD dijet background. However, we still expect some degree of discrimination for $q g$ resonance since the fraction of quark and gluon will be different for the SM background from the $q g$ resonance, where it is expected to be an admixture of 50\% quark and 50\% gluon.

Before going into the details of our results, it is useful to highlight the distributions of various event variables and jet substructure (JSS) observables. We therefore plot the distributions of some of the important event variables as well as JSS observables in Fig. 3, which shows the normalized distributions of these observables for leading $\left(j_{1}\right)$ and subleading $\left(j_{2}\right)$ jets. In all the panels of Fig. 3, the blue dashed lines represent the distributions of $g g$ resonance, while the green solid lines are for $q q$ resonance and the red dot-dashed lines are for $q g$ resonance. The normalized distributions for the SM dijet background are also shown in black dashed lines for all the variables. We note that the distributions of JSS observables for $j_{1}$ and $j_{2}$ (arranged in descending order of $p_{T}$ ) are similar for different types of signals as well as for the background. This is expected because of the following reasons. In the case of $g g(q q)$ resonance signal, both the jets are gluon-initiated (quark-initiated) jets. In the case of $q g$ resonance signal, the percentage of quark-initiated and gluon-initiated jets in $j_{1}$ is the same as in $j_{2}$ in a set of events. The same argument applies for the SM dijet background as well.

One may now proceed on to perform a cut based analysis to maximize the different types of signals with respect to the background using the shapes of the distributions. However, a multivariate analysis (MVA) further enhances the signal with respect to background by providing a classifier variable after combining all the input variables in an optimized way. In this study, we used BDT classifier variable for the rest of the analyses to follow. For the implementation of BDT, the TMVA2 . 0 [75] package which is built-in in Root $6[76,77]$ was used. At this point, we note that though the resonance masses of all the three different types of resonances have been taken to be the same $(2 \mathrm{TeV})$, the peak positions and spreads of the invariant mass distribution, as shown in Fig. 2, for different types of resonances are slightly different. Hence, in order to maximize the effect of BDT analysis, we put the following cut on the invariant mass of the different types 

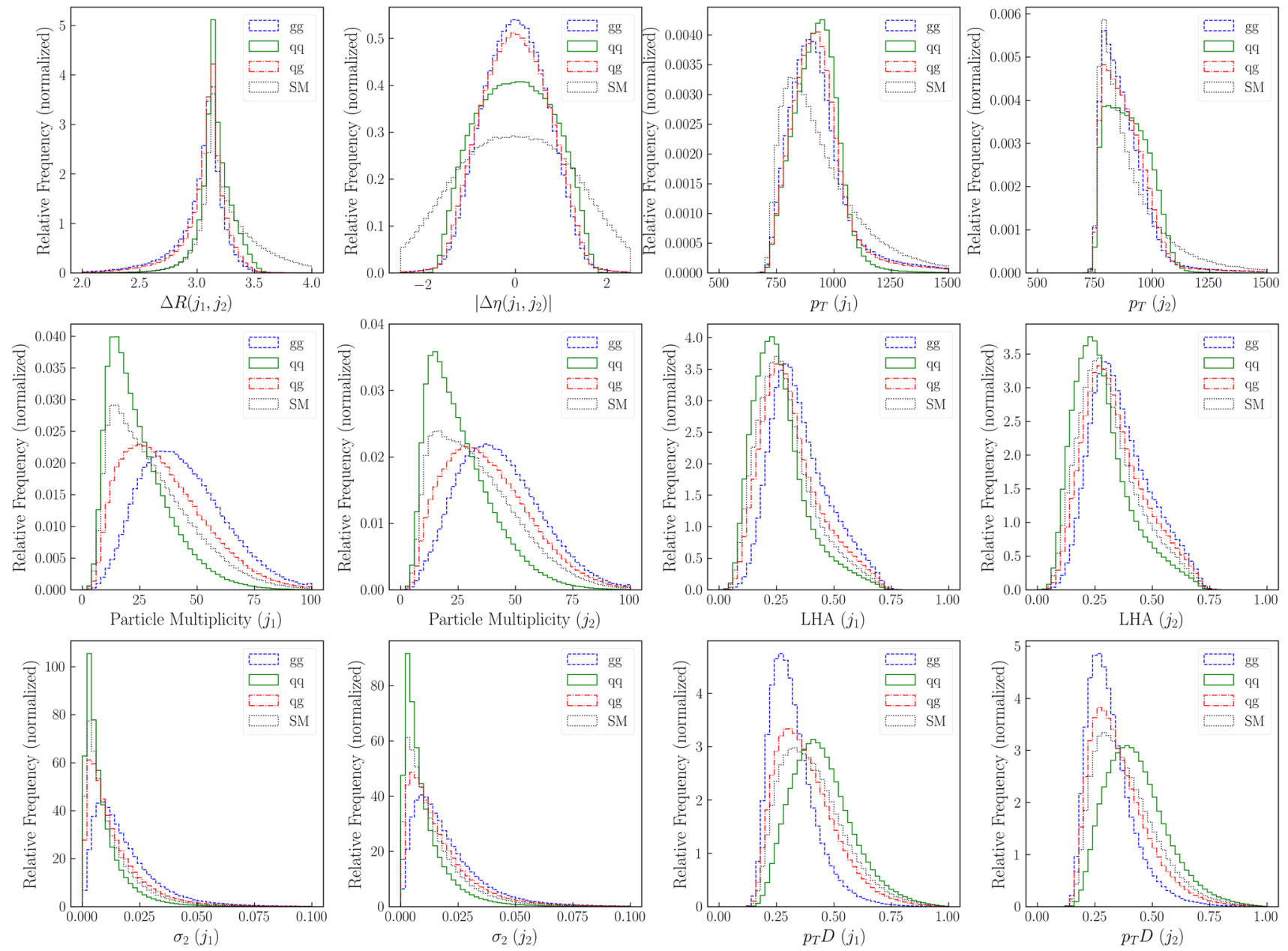

Fig. 3 Normalized distribution of jet substructure observables for the three types of signals as well as for the SM dijet background. In all the panels, blue dashed lines are for $g g$ resonance, green solid lines are for $q q$ resonance, red dot-dashed lines are for $q g$ resonance, and black dotted lines are for SM background

of resonances ${ }^{3}$ before passing them on to the BDT analysis.

$-1580<M_{g g}<2100$
$-1680<M_{q q}<2180$
$-1600<M_{q g}<2120$

As mentioned in Refs. [19,20,23,24], some jet substructure observables are better suited for quark and gluon jet tagging than others. In our work we too perform a thorough investigation of the jet substructure observables to find out

\footnotetext{
3 Note that we want the BDT to exploit more information from the JSS variables and focus on the backgrounds that are lying under the signal peak and can not be easily suppressed by looking at dijet mass only. Though we propose a full search analysis in an experiment to construct mass dependent BDT discriminators, here we present only a case study considering only a single mass point at $2 \mathrm{TeV}$. This search strategy however can be replicated for each considered mass point in a real experimental analysis.
}

which of the observables are best suited for better distinguishing power. We also note that the same jet substructure observables will be equally important in distinguishing the different types of final state events. To highlight this, we list the variable importance in BDT[75] in Table 2. The variable importance is defined as the weighted fraction of how many times a particular variable is used to split the decision tree nodes. The weight is the product of the separation gain-squared it has achieved in each split occurrence and the number of events in the node. As we see that the most important variables are the event variables, viz. $m_{j j}, \Delta R\left(j_{1}, j_{2}\right)$ and $\Delta \eta$. However, the importance of JSS observables are not very small. Combination of JSS observables give rise to substantial contribution.

In this study, our primary aim is to investigate how much improvement one can get over the current resonant dijet search by the use of jet substructure observables. Hence, we optimize BDT response in two stages. In order to see that the use of jet substructure observables help in discriminating 
Table 2 List of values of variable importance defined as the weighted fraction of the times a particular variable has been used in the boosted decision tree training. All the values are calculated in the training with JSS observables

\begin{tabular}{|c|c|c|c|c|c|c|}
\hline \multirow[t]{3}{*}{ Variables } & \multicolumn{6}{|c|}{ Variable importance (in \%) } \\
\hline & \multicolumn{2}{|l|}{$g g$} & \multicolumn{2}{|l|}{$q q$} & \multicolumn{2}{|l|}{$q g$} \\
\hline & Jet 1 & Jet 2 & Jet 1 & Jet 2 & Jet 1 & Jet 2 \\
\hline \multicolumn{7}{|l|}{ Event variables } \\
\hline$m_{j j}$ & 7.38 & & 9.41 & & 8.81 & \\
\hline$\Delta R\left(j_{1}, j_{2}\right)$ & 9.16 & & 7.03 & & 8.41 & \\
\hline$\left|\Delta \eta\left(j_{1}, j_{2}\right)\right|$ & 5.34 & & 5.67 & & 5.67 & \\
\hline$p_{T}$ & 5.70 & 4.87 & 6.24 & 8.50 & 7.68 & 6.16 \\
\hline Energy & 5.12 & 5.09 & 4.19 & 4.37 & 4.56 & 4.56 \\
\hline$\eta$ & 4.47 & 4.03 & 4.05 & 4.29 & 4.50 & 4.48 \\
\hline \multicolumn{7}{|l|}{ JSS observables } \\
\hline Particle multiplicity & 11.98 & 9.23 & 8.43 & 7.88 & 9.14 & 8.45 \\
\hline$p_{T} D$ & 6.20 & 4.87 & 6.23 & 6.07 & 5.57 & 5.55 \\
\hline LHA & 5.33 & 4.86 & 5.85 & 5.75 & 5.32 & 5.16 \\
\hline$\sigma_{2}$ & 3.11 & 3.26 & 2.86 & 3.17 & 2.91 & 3.06 \\
\hline
\end{tabular}

signal from the background, we first optimize BDT response for the signal using only event variables, viz. $p_{T}, \eta$ of both the jets, $\Delta R\left(j_{1}, j_{2}\right),|\Delta \eta|$, and $m_{j j}$ for the discrimination of signal from the SM dijet background. We then supplement the analysis by adding jet substructure (JSS) observables, viz. particle multiplicity, LHA, $p_{T} D, \sigma_{2}$ of leading as well as sub-leading jets in addition to the simple event variables. To illustrate the effect of JSS observables, we plot the distributions for BDT response for different types of signals as well as for the background in Fig. 4. In all the panels of Fig. 4, the red solid (black dashed) line represents the BDT response for the signal (background) without using the JSS observables while the green (blue) shade represents the same with the JSS observables. The responses shown are for (a) $g g$ resonance, (b) $q q$ resonance, and (c) $q g$ resonance. The improvement of signal-background separation can be understood from the relative shift in the BDT response distribution for the signal and the background in Fig. 4. As we can see from the figure that the distribution of BDT response for the signal (red solid) and that for the background (black dashed) are close to each other when JSS observables were not considered. However, after the incorporation of the JSS observables, the two distributions (green and blue shaded regions) are better separated. This tells us that the use of JSS observables will further improve the probe of the signals over the background although the degree of improvement will be different for different types of signals. A quick comparison among the BDT responses tells us that the improvement will be better for $g g$ and $q q$ resonance signals than the $q g$ signal.

Although Fig. 4 shows some degree of improvement when JSS observables are used along with event variables, it does not provide any quantitative assessment of the improvement. One of the ways to compare and see the improvement is via receiver operating characteristic (ROC) curve. A ROC curve gives the background rejection efficiency, i.e. the fraction of background rejected out of the total background for a given signal efficiency (the fraction of signal accepted out of the total signal). For a single variable, ROC curve can easily be obtained by sliding upper or lower cut on that particular variable and plotting the values of background rejection efficiency versus signal efficiency. However, for more than one variable, the ROC curve is not unique. Since the MVA already provides single optimized classifier variable from more than one input variable, we can use this classifier variable to draw
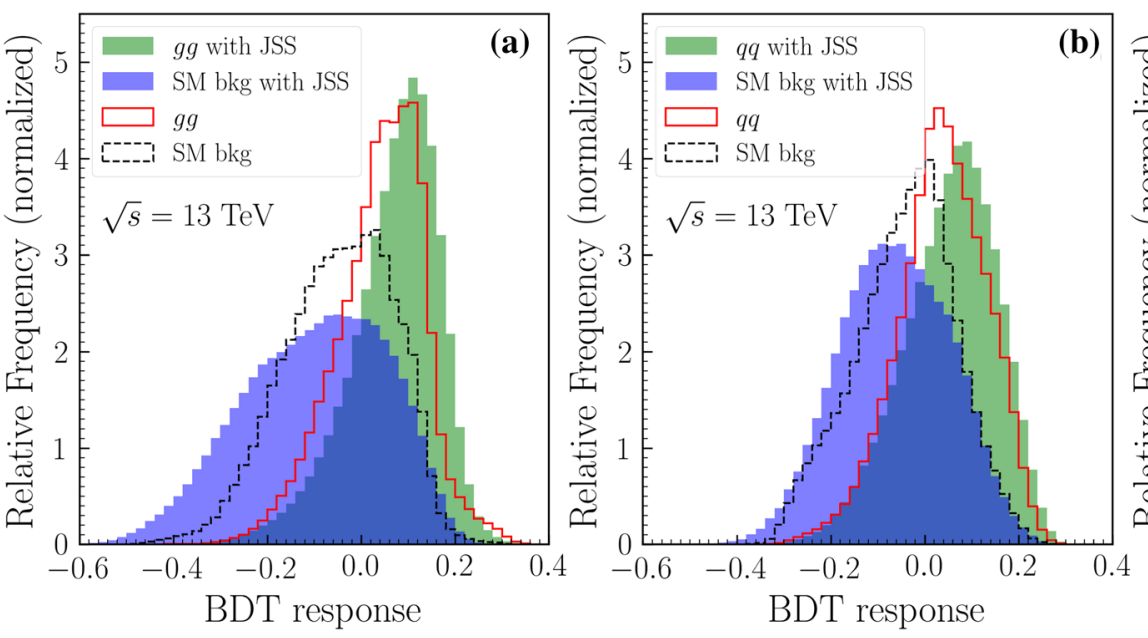

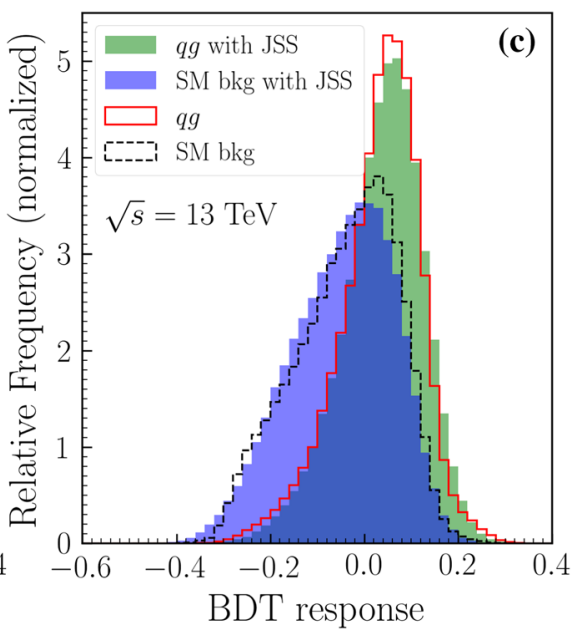

Fig. 4 BDT response for the signal and the background for three resonance cases. In all the panels, red solid (black dashed) line represents BDT response for the signal (background) without using the JSS observables while the green (blue) shade represents the same with the JSS observables. The responses shown are for a $g g$ resonance, $\mathbf{b} q q$ resonance, and $\mathbf{c} q g$ resonance 

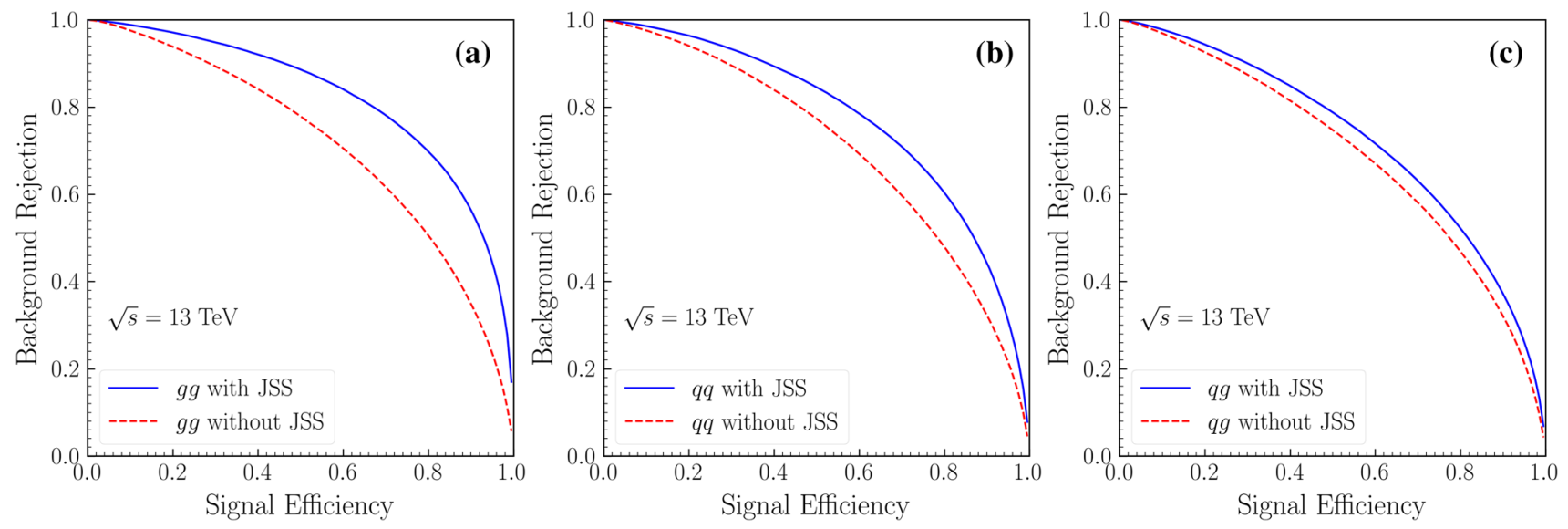

Fig. 5 Illustrating ROC curves for the a $g g$ resonance, $\mathbf{b} q q$ resonance and $\mathbf{c} q g$ resonance signal against the SM dijet background for analyses with (blue solid) and without (red dashed) JSS observables

ROC curves for the three different types signals with respect to the background. We plot the ROC curves in Fig. 5 with-JSS (blue solid) and without-JSS (red dashed) observables for all the three types of resonances. We can see, quite clearly, a significant improvement if we add JSS observables to the analysis.

Once the numbers of signal and background events are estimated, we are ready to calculate signal significance by the traditional cut and count method. In this method, one generally counts the number of signal $(S)$ and background $(B)$ events after imposing a cut at a specific point in the variable space. The cut is chosen in such a way so that the significance, defined as $S / \sqrt{B}$, is maximized. Since BDT response is already an optimized variable, this single variable can be used for cut and count analysis method. However, estimation of signal significance using the shape of the signal and background distribution of a particular variable gives significant improvement over the simple cut and count method. In this work, we performed profile likelihood ratio method to calculate signal significance. We used profile likelihood calculator, which is implemented in Roostats [78] package, after building probability distribution functions (PDF) for the signal and for the background using Number Counting Pdf Factory [79-81] from the BDT response histograms. In each bin of the histograms, the factory incorporates relative systematic uncertainty $\left(\lambda_{i}\right)$ to the fraction of events in the sideband region (defined as $\tau_{i}$ in Refs. [79-81]), as follows

$\tau_{i}=\frac{1+\sqrt{1+4 \lambda_{i}^{2}}}{2 \lambda_{i}^{2} b_{i}}$

where $b_{i}$ is the number of background and $i$ represents the index of the bin. In this analysis, the relative uncertainty in the background is taken to be flat, i.e. $\lambda_{i}=\lambda$ for all the bins. The variation of the signal significance as a function of relative systematic uncertainty $(\lambda)$ is shown in the left panel of Fig. 6 for $g g$ resonance signal. The red-dashed line is for the analysis "without-JSS" case while the blue-solid line is for "with-JSS" case. The luminosity is taken to be $100 \mathrm{fb}^{-1}$ in the signal significance calculation. From the figure, we see that the signal significance can be improved if we use JSS observables in addition to the event variable. To quantify the improvement of using JSS observables, we define a significance improvement variable as

significance improvement $=\frac{\sigma^{\mathrm{JSS}}}{\sigma}$

where $\sigma$ and $\sigma^{\text {JSS }}$ represent significances for without-JSS and with-JSS case respectively. significance improvement is plotted as a function of $\lambda$ in the right panel of Fig. 6. We can easily say that the signal significance can be improved by about $40 \%$ with the use of JSS observables even with systematic uncertainty higher than $2 \%$.

The plots in Fig. 6 consider the same values of $\lambda$ for both the with-JSS and without-JSS case and is more of a phenomenological choice. In an experimental analysis, with-JSS case may have additional source of uncertainty and therefore the plots for the individual cases should be seen with respect to the absolute systematics and not relative to each other. However, from Fig. 6, we find that with-JSS case still performs better, even with a $2 \%$ additional uncertainty. Hence, one would still benefit from the study with jet substructure once more accurate estimates of systematics is performed in the experimental analysis.

We note that we did not carry out any explicit analysis on the systematics. One of the major source of systematic uncertainty could be from the determination of jet energy scale. Though we have corrected the jet four-momenta for the jet energy scale, we did not really estimate the uncertainty in that. Another major source of uncertainty can be from PDF and parton shower scale uncertainty. Also, it is 

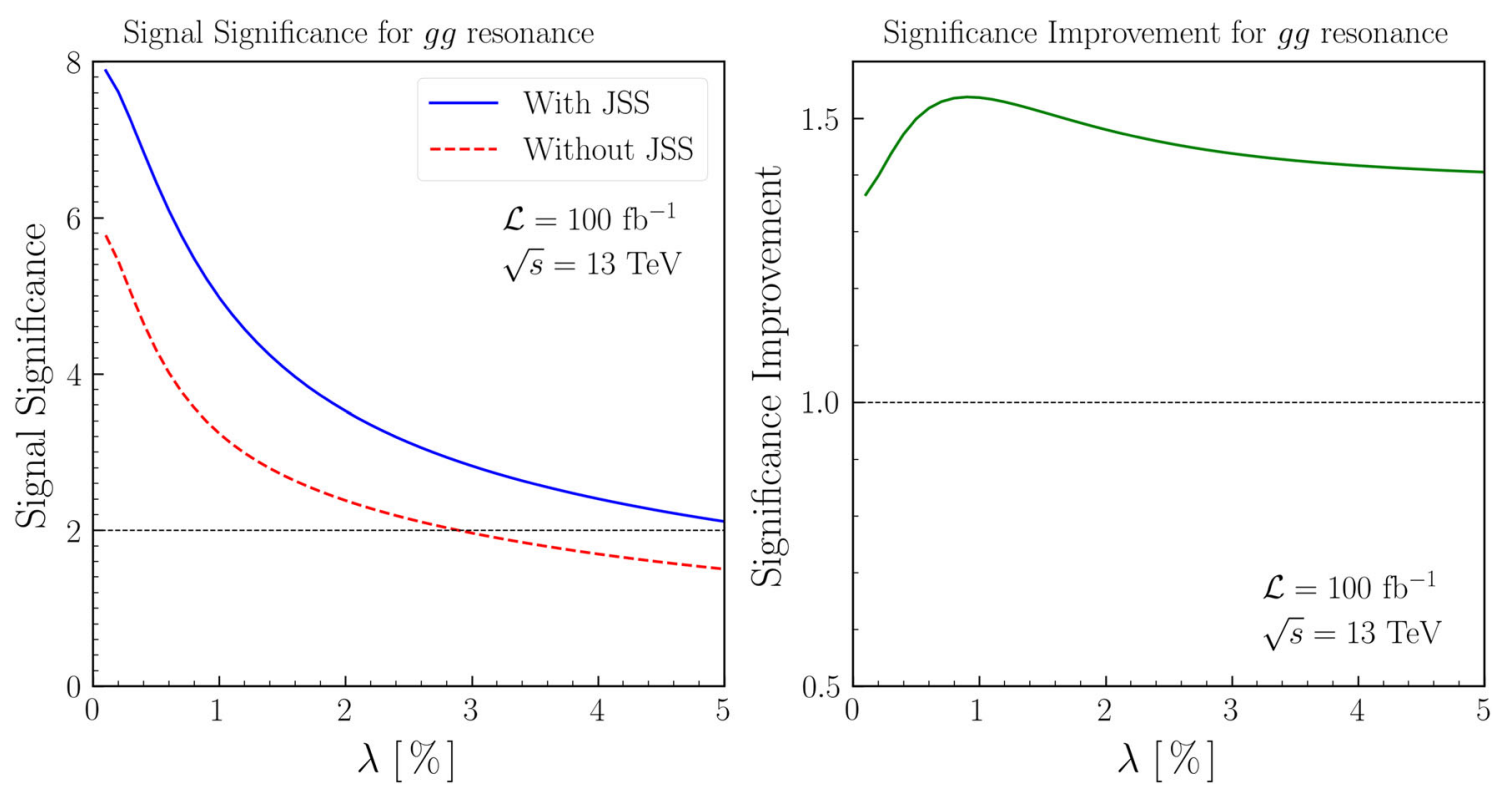

Fig. 6 (Left) variation of signal significance as a function of relative systematic uncertainty $(\lambda)$ for with-JSS (blue-solid) and without-JSS (reddashed) case. (Right) variation of significance improvement as a function of $\lambda$. The variations plotted are for $g g$ resonance signal

not guaranteed that the uncertainty would remain uniform in the whole range of signal efficiency. However, our analysis is still useful in inferring that JSS observables help improve the performance of existing dijet analysis even in the case of non-zero systematics.

Another important point to discuss is the calibration and estimation of systematic uncertainties in BDT data. The distributions of the input variables to the BDT can be validated in data in a background enriched region. The background enriched region, for example, can be considered to be the region in dijet mass distribution which are already excluded by experiments at $95 \% \mathrm{CL}$. Similarly, the distributions of BDT output can also be validated in data in such a background enriched region. A correction can be derived and/or an additional uncertainty can be assigned if any systematic shift is observed. Similarly, the systematic uncertainties affecting the input variables can be propagated to the BDT ouput by evaluating the BDT response for up and down variations of each of the input variables.

We carried out a very similar exercise (analyses) for the other two types of resonant configuration in the dijet, i.e. the $q q$ and $q g$ resonances. The results are shown in Fig. 7. In the left panel of Fig. 7, we plotted the variation of signal significance as a function of relative systematic uncertainty $(\lambda)$ for the $q q$ (top) and $q g$ (bottom) resonance signals against SM dijet background. Significance improvement as a function of $\lambda$ is plotted in the right panel of the figure. We again observe reasonable improvements as seen for $g g$ resonance. For $q g$ resonance the improvement is slightly depleted compared to the other two cases. This is because both $q g$ signal and SM dijet background has an admixture of both quark and gluon jet properties in either jet in the dijet event. Nevertheless, we could achieve some degree of improvement since the fraction of quark and gluon jets are different in the signal with respect to the background. A quantitative comparison of the upper limits on the product of cross section (partonic) and acceptance $(A)$ at $95 \%$ CL is also shown in Table 3 for a fixed value of the integrated luminosity of $36 \mathrm{fb}^{-1}$. It is worth pointing out here that we make some approximations, such as normalizing our background estimates in the relevant mass bin with that of the CMS analysis and account for approximate acceptance values $A,{ }^{4}$ to arrive at the upper limits shown in Table 3.

\section{Discrimination between $g g$ vs. $q g$ vs. $q q$ resonances}

Once the signal is identified over the SM background, we can expect to discriminate between different types of resonances, e.g. discriminating $g g$ resonance from $q q$ and $q g$ resonances. This is in similar spirit of determining the CP properties $[82,83]$ of the observed $125-\mathrm{GeV}$ scalar at the LHC. For the purpose of discriminating different types of signals, the BDT is trained for one signal against the other signal. If we consider three different types of signals, we have three different combinations, viz. (a) $g g$ vs. $q q$, (b) $g g$ vs. $q g$ and (c) $q g$ vs. $q q$. The BDT responses for these three combinations are shown in Fig. 8. Panel (a) shows BDT responses

\footnotetext{
4 The acceptance factors as provided in Ref. [2] are taken as 57\%, 85\% and $76 \%$ for $g g, q q$ and $q g$ resonances respectively. These numbers are quoted for $1.6 \mathrm{TeV}$ resonance in the CMS paper.
} 

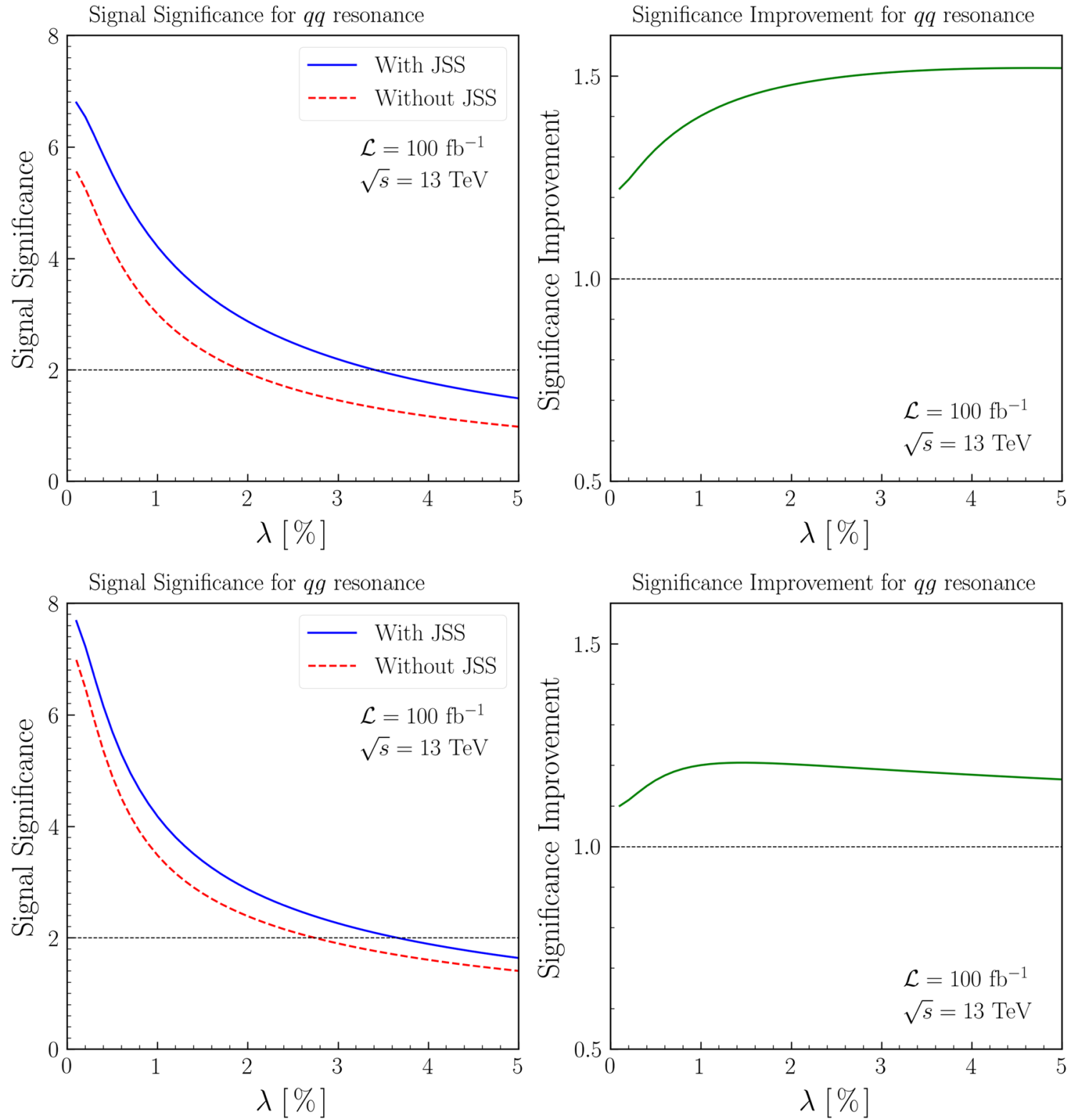

Fig. 7 (Left) variation of signal significance as a function of relative systematic uncertainty $(\lambda)$ for with-JSS (blue-solid) and without-JSS

function of $\lambda$. The variations plotted are for (top) $q q$ and (bottom) $q g$ resonance signal

Table 3 Comparison of $95 \%$ CL upper limit on $\sigma \times A$ between our analyses vs. CMS result [2] at $M=2 \mathrm{TeV}$. The upper limits are given for two different values of relative systematic uncertainty $\lambda$. The analysis is performed for $\sqrt{s}=13 \mathrm{TeV}$ and $\mathscr{L}=36 \mathrm{fb}^{-1}$

\begin{tabular}{|c|c|c|c|c|}
\hline \multirow[t]{2}{*}{ Resonance type } & \multirow{2}{*}{$\begin{array}{l}\text { Relative systematics } \\
\text { uncertainty }(\lambda)\end{array}$} & \multicolumn{2}{|c|}{ Upper limit on $\sigma \times A(\mathrm{pb})$} & \multirow{2}{*}{$\begin{array}{l}\text { CMS } \\
\text { Limit [2] }\end{array}$} \\
\hline & & With JSS & Without JSS & \\
\hline \multirow[t]{2}{*}{$g g$} & $\lambda=0 \%$ & 0.052 & 0.070 & \multirow[t]{2}{*}{0.297} \\
\hline & $\lambda=2 \%$ & 0.071 & 0.111 & \\
\hline \multirow[t]{2}{*}{$q q$} & $\lambda=0 \%$ & 0.042 & 0.052 & \multirow[t]{2}{*}{0.108} \\
\hline & $\lambda=2 \%$ & 0.058 & 0.078 & \\
\hline \multirow[t]{2}{*}{$q g$} & $\lambda=0 \%$ & 0.063 & 0.068 & \multirow[t]{2}{*}{0.167} \\
\hline & $\lambda=2 \%$ & 0.100 & 0.120 & \\
\hline
\end{tabular}



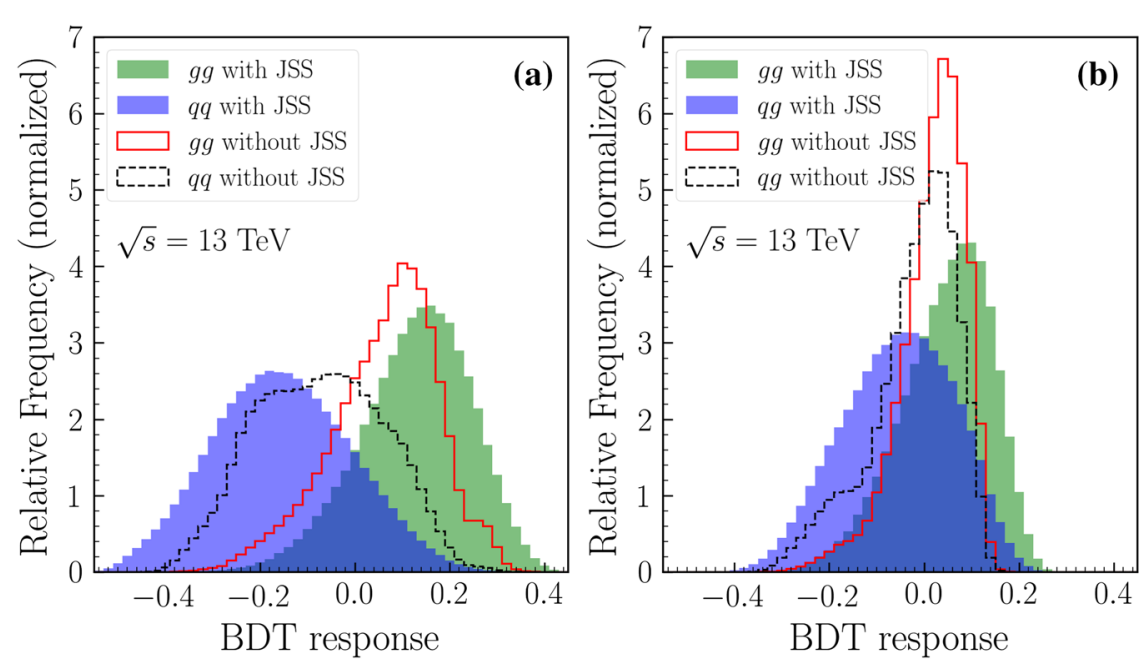

Fig. 8 Normalized distribution of BDT responses for a $g g$ vs. $q q$, b $g g$ vs. $q g$, and $\mathbf{c} q g$ vs. $q q$ signal separation. In all the panels, red solid and black dashed lines represent the classifier responses for the

for $g g$ vs. $q q$ signal separation while panels (b) and (c) show BDT responses for $g g$ vs. $q g$ signal discrimination and $q g$ vs. $q q$ discrimination respectively. In all the panels, red solid and black dashed lines represents the classifier response for the case when JSS observables are not used while green and blue shaded regions represent the case when JSS observables are used along with event observables. In this study we used the same set of event variables and JSS observables as done in the analysis of signal separation from the SM dijet background. All the panels in Fig. 8 show similar features, as seen in Fig. 4, i.e. the distributions of BDT response of two resonances for "without-JSS" case have higher overlap compared to the case when JSS observables were used. This tells us that different types of resonances can be better separated if we use JSS observables in addition to the event variables.

To get a quantitative idea, we calculated separation power [75] for the three different combinations, viz. (a) $g g$ vs. $q q$, (b) $g g$ vs. $q g$ and (c) $q g$ vs. $q q$. The separation power is defined as

$$
\left\langle S^{2}\right\rangle=\frac{1}{2} \int d x \frac{(p(x)-q(x))^{2}}{p(x)+q(x)}
$$

where $p(x)$ and $q(x)$ are normalized distribution functions for the two resonances in consideration for discrimination. The values for separation power for the BDT response in "without-JSS" and "with-JSS" analysis is listed in Table 4. We can see from Table 4 as well as from Fig. 8 that the use of JSS observables help us to discriminate between the different types of resonances much better. case when JSS observables are not used while green and blue shaded regions represent the case when JSS observables are used along with event variables

Table 4 Separation power between different resonance hypotheses for without-JSS and with-JSS case

\begin{tabular}{lll}
\hline & $\left\langle S^{2}\right\rangle$ (without-JSS) $(\%)$ & $\left\langle S^{2}\right\rangle$ (with-JSS) (\%) \\
\hline$g g$ vs. $q q$ & 23.8 & 58.0 \\
$g g$ vs. $q g$ & 5.7 & 17.8 \\
$q g$ vs. $q q$ & 11.4 & 32.2 \\
\hline
\end{tabular}

\section{Summary and outlook}

To summarize, we studied dijet resonances at the LHC and look at the application of jet substructure techniques to such resonances. These resonances may carry different partonic imprints in the jets which will be driven by the spin and color structure of the on-shell particle produced as a resonance. We note that JSS techniques have become an essential part of today's collider physics and are being utilized as a very effective tool to understand physics at high energy colliders. Our aim of using jet substructure in this work is to make a statement on the improvements one can achieve in resonant search strategies at the colliders in the dijet final state. However, such an improvement is not restricted to only an exclusive dijet final state but can be applied when final states are of multijet in nature.

Currently, experimental collaborations put 95\% C.L. upper limit on the production cross section of different types of resonances, viz. $g g, q q$, and $q g$ resonances using only lineshape information of these types of resonances at $13 \mathrm{TeV}$ collider. In this work, we attempt to make use of JSS to improve the existing search strategies of heavy colored resonances in dijet channel. We improve upon earlier studies by perform- 
ing a more realistic event analysis by including fast detector simulation and jet energy correction. We show how the jet substructure observables help in discriminating a signal for any of the heavy dijet resonances, viz. $g g, q q$, and $q g$, from the SM QCD dijet background. We utilize BDT multivariate classifier for the discrimination. We highlight our results in the form of ROC curves where we find considerable improvement in the ROC curves when we use jet substructure observables in addition to the simple event variables. We also find that the improvement in signal significance is substantial even in the presence of non-zero systematic uncertainties. This suggests that, with the help of jet substructure technique, the search for different types of heavy resonances can be improved to a great extent at the LHC. Furthermore, we also establish and show that distinction between different types of resonances can also be achieved to a higher degree if jet substructure observables are used in addition to the event variables. The same technique can also be effectively applied to proposed future high energy machine although analyses with only $13 \mathrm{TeV}$ has been presented in this article.

Acknowledgements TS and SKR acknowledge financial support from the Department of Atomic Energy, Government of India, for the Regional Centre for Accelerator-based Particle Physics (RECAPP), Harish-Chandra Research Institute. TS would like to thank Tanmoy Mondal for useful discussion. The authors would also like to thank Mrinal Dasgupta, and Satyaki Bhattacharya for their insightful lectures on jet substructure and multivariate techniques respectively, during HEP Activity Weeks 2019 organized at Harish-Chandra Research Institute.

Data Availability Statement This manuscript has no associated data or the data will not be deposited. [Authors' comment: This a theoretical study and no experimental data has been used.]

Open Access This article is licensed under a Creative Commons Attribution 4.0 International License, which permits use, sharing, adaptation, distribution and reproduction in any medium or format, as long as you give appropriate credit to the original author(s) and the source, provide a link to the Creative Commons licence, and indicate if changes were made. The images or other third party material in this article are included in the article's Creative Commons licence, unless indicated otherwise in a credit line to the material. If material is not included in the article's Creative Commons licence and your intended use is not permitted by statutory regulation or exceeds the permitted use, you will need to obtain permission directly from the copyright holder. To view a copy of this licence, visit http://creativecomm ons.org/licenses/by/4.0/.

Funded by SCOAP ${ }^{3}$.

\section{References}

1. N. Arkani-Hamed, T. Han, M. Mangano, L.T. Wang, Phys. Rep. 652, 1 (2016). https://doi.org/10.1016/j.physrep.2016.07.004

2. A.M. Sirunyan et al., JHEP 08, 130 (2018). https://doi.org/10.1007/ JHEP08(2018) 130

3. A.M. Sirunyan et al., Phys. Lett. B 769, 520 (2017). https://doi. org/10.1016/j.physletb.2017.09.029,10.1016/j.physletb.2017.02. 012 [Erratum: Phys. Lett. B 772, 882 (2017)]
4. V. Khachatryan et al., Phys. Rev. Lett. 116(7), 071801 (2016). https://doi.org/10.1103/PhysRevLett.116.071801

5. V. Khachatryan et al., Phys. Rev. Lett. 117(3), 031802 (2016). https://doi.org/10.1103/PhysRevLett.117.031802

6. V. Khachatryan et al., Phys. Rev. D 91(5), 052009 (2015). https:// doi.org/10.1103/PhysRevD.91.052009

7. S. Chatrchyan et al., Phys. Rev. D 87(11), 114015 (2013). https:// doi.org/10.1103/PhysRevD.87.114015

8. M. Aaboud et al., Phys. Rev. Lett. 121(8), 081801 (2018). https:// doi.org/10.1103/PhysRevLett.121.081801

9. M. Aaboud et al., Phys. Rev. D 96(5), 052004 (2017). https://doi. org/10.1103/PhysRevD.96.052004

10. G. Aad et al., Phys. Lett. B 754, 302 (2016). https://doi.org/10. 1016/j.physletb.2016.01.032

11. J. Merten et al., Astrophys. J. 806(1), 4 (2015). https://doi.org/10. 1088/0004-637X/806/1/4

12. R.M. Harris, K. Kousouris, Int. J. Mod. Phys. A 26, 5005 (2011). https://doi.org/10.1142/S0217751X11054905

13. A.M. Sirunyan et al., JHEP 01, 097 (2018). https://doi.org/10.1007/ JHEP01(2018)097

14. A.M. Sirunyan et al., Phys. Rev. D $100(11), 112007$ (2019). https:// doi.org/10.1103/PhysRevD.100.112007

15. A.M. Sirunyan et al., Phys. Rev. Lett. 123(23), 231803 (2019). https://doi.org/10.1103/PhysRevLett.123.231803

16. A.M. Sirunyan et al., Phys. Rev. Lett. 119(11), 111802 (2017). https://doi.org/10.1103/PhysRevLett.119.111802

17. M. Aaboud et al., Phys. Lett. B 795, 56 (2019). https://doi.org/10. 1016/j.physletb.2019.03.067

18. A.J. Larkoski, E.M. Metodiev, JHEP 10, 014 (2019). https://doi. org/10.1007/JHEP10(2019)014

19. J. Gallicchio, M.D. Schwartz, JHEP 04, 090 (2013). https://doi. org/10.1007/JHEP04(2013)090

20. P. Gras, S. Höche, D. Kar, A. Larkoski, L. Lönnblad, S. Plätzer, A. Siódmok, P. Skands, G. Soyez, J. Thaler, JHEP 07, 091 (2017). https://doi.org/10.1007/JHEP07(2017)091

21. G. Aad et al., Eur. Phys. J. C 74(8), 3023 (2014). https://doi.org/ 10.1140/epjc/s10052-014-3023-z

22. Discrimination of Light Quark and Gluon Jets in $p p$ collisions at $\sqrt{s}=8 \mathrm{TeV}$ with the ATLAS Detector. Technical report ATLAS-CONF-2016-034, CERN, Geneva (2016). http://cds.cern. $\mathrm{ch} / \mathrm{record} / 2200202$. Accessed 24 Dec 2020

23. Performance of quark/gluon discrimination in $8 \mathrm{TeV}$ pp data. Technical report CMS-PAS-JME-13-002, CERN, Geneva (2013). http:// cds.cern.ch/record/1599732. Accessed 24 Dec 2020

24. Jet algorithms performance in $13 \mathrm{TeV}$ data. Technical report CMS-PAS-JME-16-003, CERN, Geneva (2017). http://cds.cern. ch/record/2256875. Accessed 24 Dec 2020

25. Performance of quark/gluon discrimination in $13 \mathrm{TeV}$ data. Technical report (2016). https://cds.cern.ch/record/2234117. Accessed 24 Dec 2020

26. S. Bright-Thonney, B. Nachman, JHEP 03, 098 (2019). https://doi. org/10.1007/JHEP03(2019)098

27. B. Bhattacherjee, S. Mukhopadhyay, M.M. Nojiri, Y. Sakaki, B.R. Webber, JHEP 01, 044 (2017). https://doi.org/10.1007/ JHEP01(2017)044

28. J. Gallicchio, M.D. Schwartz, Phys. Rev. Lett. 107, 172001 (2011). https://doi.org/10.1103/PhysRevLett.107.172001

29. Y. Sakaki, Phys. Rev. D 99(11), 114012 (2019). https://doi.org/10. 1103/PhysRevD.99.114012

30. P.T. Komiske, E.M. Metodiev, M.D. Schwartz, JHEP 01, 110 (2017). https://doi.org/10.1007/JHEP01(2017)110

31. E.M. Metodiev, B. Nachman, J. Thaler, JHEP 10, 174 (2017). https://doi.org/10.1007/JHEP10(2017)174

32. T. Cheng, Comput. Softw. Big Sci. 2(1), 3 (2018). https://doi.org/ 10.1007/s41781-018-0007-y 
33. H. Luo, M.X. Luo, K. Wang, T. Xu, G. Zhu, Sci. China Phys. Mech. Astron. 62(9), 991011 (2019). https://doi.org/10.1007/ s11433-019-9390-8

34. K. Fraser, M.D. Schwartz, JHEP 10, 093 (2018). https://doi.org/ 10.1007/JHEP10(2018)093

35. G. Kasieczka, N. Kiefer, T. Plehn, J.M. Thompson, Sci. Post Phys. 6, 069 (2019). https://doi.org/10.21468/SciPostPhys.6.6.069

36. Quark versus Gluon Jet Tagging Using Jet Images with the ATLAS Detector. Technical report ATL-PHYS-PUB-2017-017, CERN, Geneva (2017). https://cds.cern.ch/record/2275641. Accessed 24 Dec 2020

37. J.S.H. Lee, S.M. Lee, Y. Lee, I. Park, I.J. Watson, S. Yang, J. Korean Phys. Soc. 75(9), 652 (2019). https://doi.org/10.3938/jkps.75.652

38. S. Ozturk, Adv. High Energy Phys. 2014, 719216 (2014). https:// doi.org/10.1155/2014/719216

39. R. Sekhar Chivukula, E.H. Simmons, N. Vignaroli, Phys. Rev. D 91(5), 055019 (2015). https://doi.org/10.1103/PhysRevD.91. 055019

40. E. Simmons, R. Chivukula, P. Ittisamai, N. Vignaroli, Separating Dijet Resonances Using the Color Discriminant Variable (WSP, 2018), pp. 235-249. https://doi.org/10.1142/ 9789813231467_0032

41. D. Karabacak, S. Nandi, S.K. Rai, Phys. Rev. D 85, 075011 (2012). https://doi.org/10.1103/PhysRevD.85.075011

42. D.A. Dicus, D. Karabacak, S. Nandi, S.K. Rai, Phys. Rev. D 87(1), 015023 (2013). https://doi.org/10.1103/PhysRevD.87.015023

43. K. Das, S. Majhi, S.K. Rai, A. Shivaji, JHEP 10, 122 (2015). https:// doi.org/10.1007/JHEP10(2015)122

44. T. Han, I. Lewis, Z. Liu, JHEP 12, 085 (2010). https://doi.org/10. 1007/JHEP12(2010)085

45. C.T. Hill, Phys. Lett. B 266, 419 (1991). https://doi.org/10.1016/ 0370-2693(91)91061-Y

46. C.T. Hill, S.J. Parke, Phys. Rev. D 49, 4454 (1994). https://doi.org/ 10.1103/PhysRevD.49.4454

47. R.S. Chivukula, A.G. Cohen, E.H. Simmons, Phys. Lett. B 380, 92 (1996). https://doi.org/10.1016/0370-2693(96)00464-9

48. C.T. Hill, E.H. Simmons, Phys. Rep. 381, 235 (2003). https://doi.org/10.1016/S0370-1573(03)00140-6. [Erratum: Phys. Rept.390,553(2004)]

49. M.I. Gresham, M.B. Wise, Phys. Rev. D 76, 075003 (2007). https:// doi.org/10.1103/PhysRevD.76.075003

50. M. Gerbush, T.J. Khoo, D.J. Phalen, A. Pierce, D. TuckerSmith, Phys. Rev. D 77, 095003 (2008). https://doi.org/10.1103/ PhysRevD.77.095003

51. N. Cabibbo, L. Maiani, Y. Srivastava, Phys. Lett. 139B, 459 (1984). https://doi.org/10.1016/0370-2693(84)91850-1

52. A. De Rujula, L. Maiani, R. Petronzio, Phys. Lett. 140B, 253 (1984). https://doi.org/10.1016/0370-2693(84)90930-4

53. J.H. Kuhn, P.M. Zerwas, Phys. Lett. 147B, 189 (1984). https://doi. org/10.1016/0370-2693(84)90618-X

54. U. Baur, I. Hinchliffe, D. Zeppenfeld, Int. J. Mod. Phys. A 2, 1285 (1987). https://doi.org/10.1142/S0217751X87000661

55. U. Baur, M. Spira, P.M. Zerwas, Phys. Rev. D 42, 815 (1990). https://doi.org/10.1103/PhysRevD.42.815

56. J.R. Andersen, et al., (2016). http://lss.fnal.gov/archive/2016/conf/ fermilab-conf-16-175-ppd-t.pdf. Accessed 24 Dec 2020

57. S. Catani, G. Turnock, B.R. Webber, Phys. Lett. B 295, 269 (1992). https://doi.org/10.1016/0370-2693(92)91565-Q

58. P.E.L. Rakow, B.R. Webber, Nucl. Phys. B 191, 63 (1981). https:// doi.org/10.1016/0550-3213(81)90286-8

59. R.K. Ellis, B.R. Webber, Conf. Proc. C860623, 74 (1986)

60. A.J. Larkoski, G.P. Salam, J. Thaler, JHEP 06, 108 (2013). https:// doi.org/10.1007/JHEP06(2013)108

61. J. Gallicchio, M.D. Schwartz, Phys. Rev. Lett. 105, 022001 (2010). https://doi.org/10.1103/PhysRevLett.105.022001
62. C. Degrande, C. Duhr, B. Fuks, D. Grellscheid, O. Mattelaer, T. Reiter, Comput. Phys. Commun. 183, 1201 (2012). https://doi.org/ 10.1016/j.cpc.2012.01.022

63. N.D. Christensen, C. Duhr, Comput. Phys. Commun. 180, 1614 (2009). https://doi.org/10.1016/j.cpc.2009.02.018

64. A. Alloul, N.D. Christensen, C. Degrande, C. Duhr, B. Fuks, Comput. Phys. Commun. 185, 2250 (2014). https://doi.org/10.1016/j. cpc.2014.04.012

65. J. Alwall, R. Frederix, S. Frixione, V. Hirschi, F. Maltoni, O. Mattelaer, H.S. Shao, T. Stelzer, P. Torrielli, M. Zaro, JHEP 07, 079 (2014). https://doi.org/10.1007/JHEP07(2014)079

66. T. Sjöstrand, S. Ask, J.R. Christiansen, R. Corke, N. Desai, P. Ilten, S. Mrenna, S. Prestel, C.O. Rasmussen, P.Z. Skands, Comput. Phys. Commun. 191, 159 (2015). https://doi.org/10.1016/j.cpc.2015.01. 024

67. J. de Favereau, C. Delaere, P. Demin, A. Giammanco, V. Lemaître, A. Mertens, M. Selvaggi, JHEP 02, 057 (2014). https://doi.org/10. 1007/JHEP02(2014)057

68. M. Cacciari, G.P. Salam, G. Soyez, Eur. Phys. J. C 72, 1896 (2012). https://doi.org/10.1140/epjc/s10052-012-1896-2

69. D. Krohn, J. Thaler, L.T. Wang, JHEP 02, 084 (2010). https://doi org/10.1007/JHEP02(2010)084

70. S.D. Ellis, C.K. Vermilion, J.R. Walsh, Phys. Rev. D 80, 051501 (2009). https://doi.org/10.1103/PhysRevD.80.051501

71. S.D. Ellis, C.K. Vermilion, J.R. Walsh, Phys. Rev. D 81, 094023 (2010). https://doi.org/10.1103/PhysRevD.81.094023

72. J.M. Butterworth, A.R. Davison, M. Rubin, G.P. Salam, Phys. Rev. Lett. 100, 242001 (2008). https://doi.org/10.1103/PhysRevLett. 100.242001

73. A.J. Larkoski, S. Marzani, G. Soyez, J. Thaler, JHEP 05, 146 (2014). https://doi.org/10.1007/JHEP05(2014)146

74. G. Aad et al., Eur. Phys. J. C 73(3), 2304 (2013). https://doi.org/ 10.1140/epjc/s10052-013-2304-2

75. A. Hoecker, P. Speckmayer, J. Stelzer, J. Therhaag, E. von Toerne, H. Voss, M. Backes, T. Carli, O. Cohen, A. Christov, et al., TMVA - Toolkit for Multivariate Data Analysis. CERNOPEN-2007-007. http://cds.cern.ch/record/1019880

76. R. Brun, F. Rademakers, Nucl. Instrum. Methods A389, 81 (1997). https://doi.org/10.1016/S0168-9002(97)00048-X

77. F. Rademakers, P. Canal, A. Naumann, O. Couet, L. Moneta, V. Vassilev, D. Piparo, G. GANIS, B. Bellenot, S. Linev, wverkerke, P. Mato, E. Guiraud, TimurP, G. Amadio, M. Tadel, wlav, A. Gheata, S. Roiser, marsupial, E. Tejedor, A. Bose, CristinaCristescu, R. Isemann, X. Valls, O. Zapata, K. Albertsson, A. Eie, H. Voss, B.P. Bockelman, Root-project/root: first release of the v616 series. (2019). https://doi.org/10.5281/zenodo.2557526

78. L. Moneta, K. Belasco, K.S. Cranmer, S. Kreiss, A. Lazzaro, D Piparo, G. Schott, W. Verkerke, M. Wolf, PoS ACAT2010, 057 (2010). https://doi.org/10.22323/1.093.0057

79. K. Cranmer, in Statistical Problems in Particle Physics. Astrophysics and Cosmology, pp. 112-123 (2005). https://doi.org/10. 1142/9781860948985_0026

80. K.S. Cranmer, (2008). https://doi.org/10.5170/CERN-2008-001. 47. http://www.cds.cern.ch/record/1099969

81. R.D. Cousins, J.T. Linnemann, J. Tucker, Nucl. Instrum. Methods A 595(2), 480 (2008). https://doi.org/10.1016/j.nima.2008.07.086

82. G. Aad et al., Phys. Rev. Lett. 125(6), 061802 (2020). https://doi. org/10.1103/PhysRevLett.125.061802

83. A.M. Sirunyan et al., Phys. Rev. Lett. 125(6), 061801 (2020). https://doi.org/10.1103/PhysRevLett.125.061801 